Article

\title{
Glycosylation of a Newly Functionalized Orthoester Derivative
}

\author{
Kohei Kawa, Tsuyoshi Saitoh, Eisuke Kaji and Shigeru Nishiyama * \\ Department of Chemistry, Faculty of Science and Technology, Keio University, Hiyoshi 3-14-1, \\ Kohoku-ku, Yokohama 223-8522, Japan
}

* Author to whom correspondence should be addressed; E-Mail: nisiyama@chem.keio.ac.jp;

Tel./Fax: +81-45-566-1717.

Received: 26 January 2014; in revised form: 20 February 2014 / Accepted: 21 February 2014 /

Published: 24 February 2014

\begin{abstract}
Tandem glycosylation of the 6-O-Fmoc-substituted benzyl orthoester derivative 2a was carried out in moderate yields by electrogenerated acid (EGA). The Fmoc group was effectively removed under mild basic conditions, and the product was submitted to the subsequent glycosylation.
\end{abstract}

Keywords: glycosylation; orthoester; electrogenerated acid (EGA); Fmoc group; selective deprotection

\section{Introduction}

With the discovery of the numerous biologically important roles of sugar chains, a number of glycosylation protocols have been reported [1,2]. Generally, the glycosylation process consists of efficient generation of an oxocarbenium ion species as a glycosyl donor and modulation of its selective nucleophilic attack on aglycons. Enhancement of $\beta$-selectivity is supported by the participation of acyl-protected hydroxyl groups. In particular, construction of a cyclic carboxonium ion between the $\mathrm{C} 1$ and $\mathrm{C} 2$ positions is an effective method to obtain the corresponding $\beta$-glycosidic linkage of D-sugars, although orthoesters are produced as side products when sterically hindered functional groups are used in glycosyl donors and acceptors [3-6]. Orthoesters were known to be converted to the corresponding glycosides under acidic conditions, and relatively stable derivatives were employed as glycoside precursors by Kochetkov [7-11] and Kunz [12,13]. We reported the synthesis and reaction of the orthoester 1 (Figure 1), which could be purified by silica gel column chromatography and kept for one month at room temperature [14]. Upon glycosylation with appropriate alcohols in the presence of the 
electrogenerated acid (EGA) [15-20], which is considered to be anhydrous $\mathrm{HClO}_{4}$ produced by anodic oxidation of cyclohexanol and $\mathrm{Bu}_{4} \mathrm{NClO}_{4}$, the corresponding glycosides were produced in high (primary $\mathrm{OH}$ ) to moderate (secondary $\mathrm{OH}$ ) yields, even on tertiary $\mathrm{OH}$ groups [14]. Upon comparison with Lewis acids and Brønsted acids, EGA provided better results in glycosylation reactions. To synthesize sugar chains by repeated glycosylation, devices providing effective activation of the anomeric center and the subsequent selective deprotection of an appropriate hydroxyl group in sugar units are required. The orthoester 1 was modified to examine its applicability to practical tandem glycosylation. Here, we describe a synthesis of the orthoester derivative 2a carrying an Fmoc group at the C-6 position, and its glycosylation reaction.

Figure 1. The benzyl orthoesters 1-4.
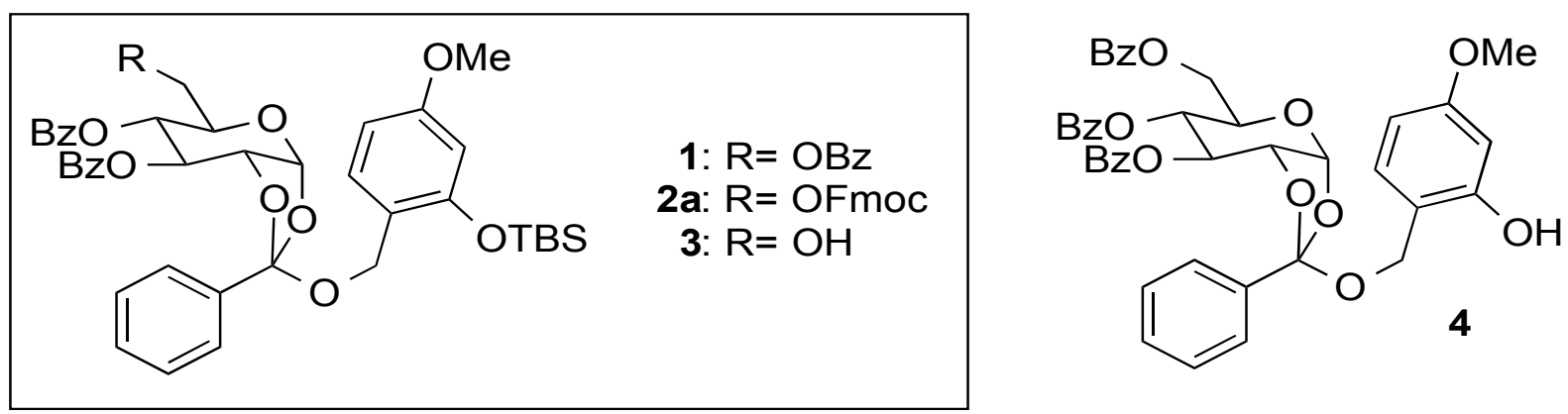

\section{Results and Discussion}

To examine the chemical properties of the orthoester $\mathbf{1}$ for the modulation of protecting groups, the acyl protecting groups were removed under basic conditions to give an unstable triol. Although removal of the TBS group in the benzylic region provided the stable phenol derivative 4 [14], the triol was labile under standard work-up conditions, and only detected by ESI mass spectrum. These observations prompted us to synthesize the orthoester derivative $\mathbf{2 a}$, in which a protecting group at the C-6 position can be selectively removed. After assessment of various protecting groups, we adopted the Fmoc group, which is removed under mild basic conditions.

\subsection{Synthesis of the Orthoester 2a}

Synthesis of the C-6 modified orthoester 2a commenced with conversion of 6- $O$-trityl-1,2,3,4-tetra$O$-benzoyl- $\beta$-D-glucopyranose, which was synthesized by the standard procedure from D-glucose, into 6-O-Fmoc-2,3,4-tri-O-benzoyl- $\alpha$-D-glucopyranosyl bromide (5) using a two-step manipulation, followed by the anomeric bromination (Scheme 1). Glycosylation of $\mathbf{5}$ with the benzyl alcohol $\mathbf{6}$ under Königs-Knorr reaction conditions provided the expected orthoester 2a (50\%) [21], along with the corresponding benzyl glycoside $\mathbf{2 b}$ (30\%). Stability of $\mathbf{2 a}$ under standard work-up and chromatographic conditions was similar to the previously reported derivative $\mathbf{1}$. The Fmoc group in 2a was selectively removed under basic conditions to give $6-\mathrm{OH}$ orthoester $\mathbf{3}$, which will be used as a glycosyl acceptor under neutral glycosylation conditions. 
Scheme 1. Synthesis of the orthoester derivative 2 a.

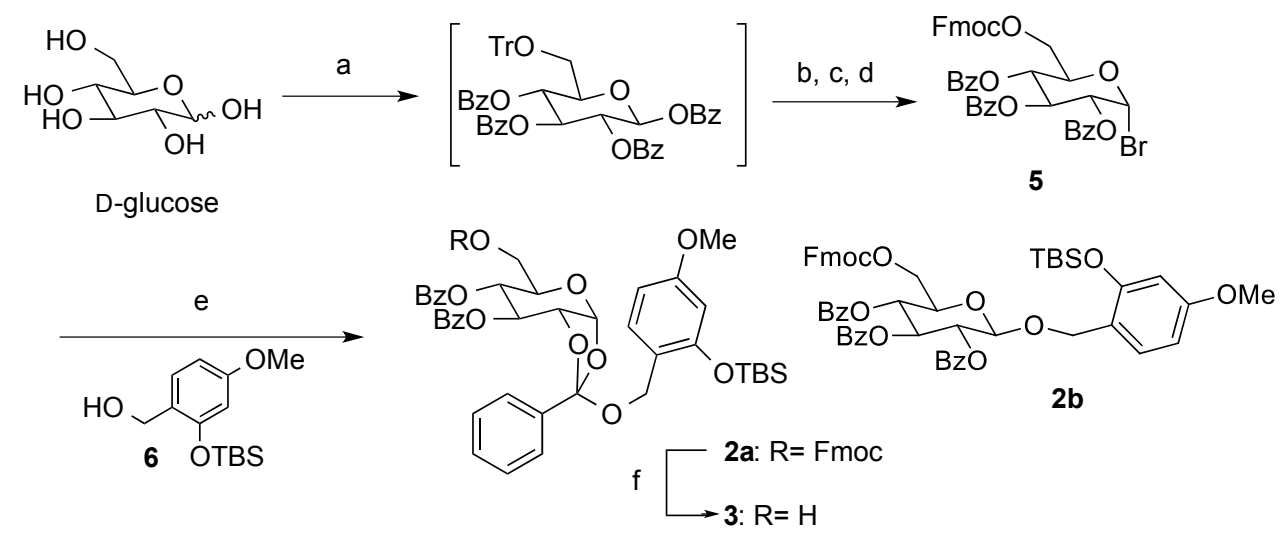

Reagents and yields: (a) $\mathrm{TrCl}, \mathrm{Et}_{3} \mathrm{~N}$, pyr.; $\mathrm{BzCl}$. (b) $\mathrm{H}_{2} \mathrm{Pd} / \mathrm{C}$. (c) $\mathrm{FmocCl}$, pyr. (d) $\mathrm{HBr}-\mathrm{AcOH}, \mathrm{Ac}_{2} \mathrm{O}, \mathrm{AcOH}\left(43 \%\right.$ in four steps. (e) $\mathbf{6}, \mathrm{Hg}(\mathrm{CN})_{2}, 4 \AA \mathrm{MS}(\mathbf{2 a}$ : $50 \%, 2 \mathrm{~b}: 30 \%)$. (f) pyr. (81\%).

\subsection{Glycosylation of the Orthoester $\mathbf{2 a}$}

When the orthoester 2a was submitted to reaction with cyclohexanol in the presence of EGA [14] and $4 \AA \mathrm{MS}$ in $1,2-\mathrm{DCM}$ at $40{ }^{\circ} \mathrm{C}$, the expected $\beta$-glycoside 7 was obtained in $62 \%$ yield (Scheme 2). Next selective removal of the Fmoc group in 7 under basic conditions was carried out to give 8 in $95 \%$ yield, without any acyl migration from C-4 to C-6 position or removal of the acyl group. Repeated glycosylation of $\mathbf{8}$ with $\mathbf{2 a}$ under the same conditions as mentioned above, gave the disaccharide $\mathbf{9}$ in $38 \%$ yield. Alternatively, glycosylation of $\mathbf{1 0}$ with $\mathbf{2 a}$ provided the disaccharide $\mathbf{1 1}$ in $53 \%$ yield. After removal of the Fmoc group, further glycosylation with 2a provided the corresponding trisaccharide 12 [22].

Scheme 2. Glycosylation reactions of the orthoester derivative 2a.

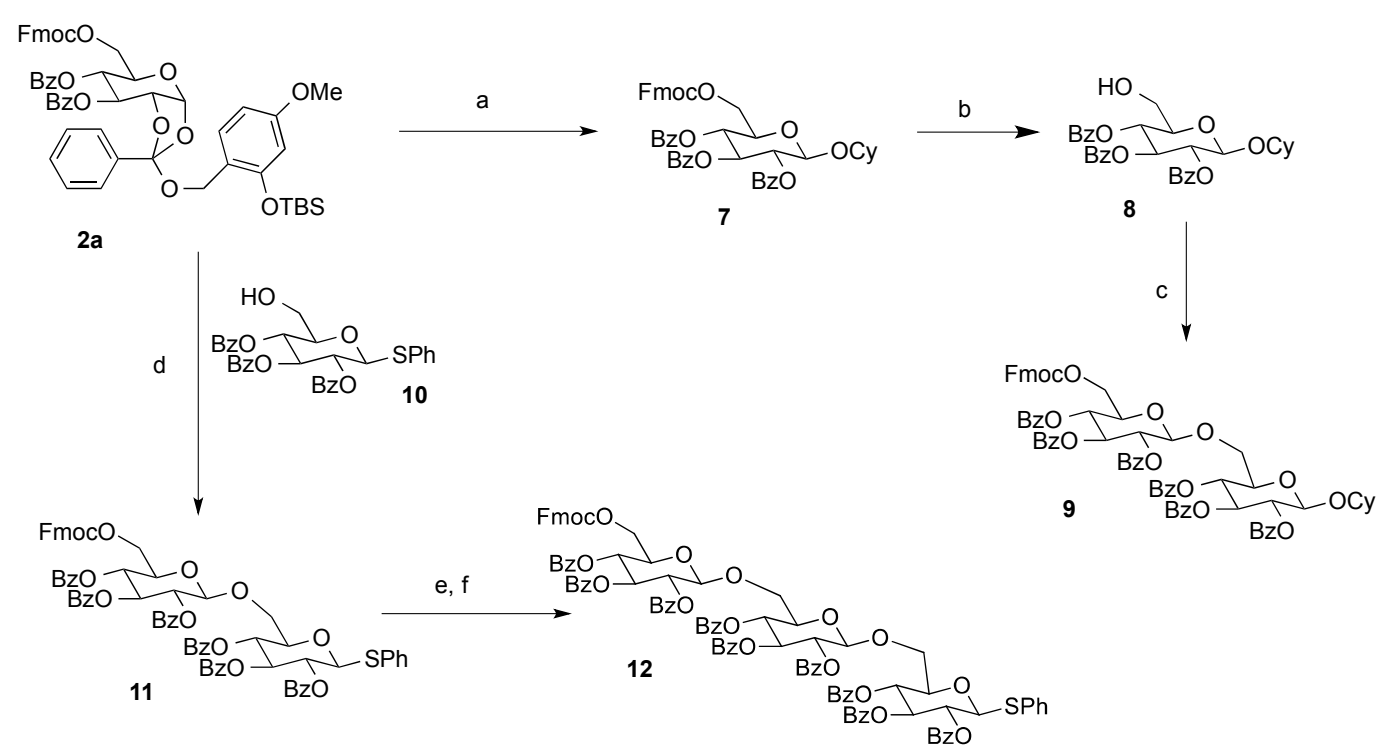

Reagents and yields: (a) cyclohexanol, EGA, $4 \AA$ MS (62\%). (b) $\mathrm{Et}_{3} \mathrm{~N}$, pyr. (95\%).

(c) 2a, EGA, $4 \AA \mathrm{MS}(38 \%)$. (d) 10, EGA, $4 \AA \mathrm{MS}(63 \%)$. (e) $\mathrm{Et}_{3} \mathrm{~N}$, pyr. (82\%).

(f) 2a, EGA, $4 \AA \mathrm{MS}(12 \%)$. 


\section{Experimental}

\section{General Information}

All reactions were carried out under an argon atmosphere unless otherwise noted. When necessary, solvents were dried prior to use. Dry THF, dry $\mathrm{Et}_{2} \mathrm{O}$ and dry $\mathrm{CH}_{2} \mathrm{Cl}_{2}$ were purchased from Kanto Chemical Co., Inc. (Tokyo, Japan). Optical rotations were measured on a JASCO P-2200 digital polarimeter with a sodium (D line) lamp. IR spectra were recorded on a JASCO Model A-202 spectrophotometer. ${ }^{1} \mathrm{H}-\mathrm{NMR}$ spectra and ${ }^{13} \mathrm{C}-\mathrm{NMR}$ spectra were obtained on JEOL JNM-GX400, JNM- $\alpha 400$, JNM-AL400 or JNM-ECX400 spectrometers in deuterated solvent using tetramethylsilane as an internal standard. Deuteriochloroform was used as a solvent, unless otherwise stated. Highresolution mass spectra were obtained on a Waters LCT Premier XE (ESI) or JEOL JMS-700 (FAB). Preparative and analytical TLC were carried out on silica gel plate (Kieselgel 60 F254, E. Merck AG., Darmstadt, Germany) using UV light, $1 \mathrm{M}$ aq. sulfuric acid, and/or 5\% molybdophosphoric acid in ethanol for detection. Kanto silica $60 \mathrm{~N}$ (spherical, neutral, 105-210 $\mu \mathrm{m}$ ) was used for column chromatography. All anodic oxidation was conducted using HA-151A (Hokuto Denko, Tokyo, Japan) as a potentiostat and galvanostat, using glassy carbon plate as anodes, and platinum plate or wire as cathode.

2,3,4-Tri-O-benzoyl-6-O-9-fluorenylmethyloxycarboxyl- $\alpha$-D-glucopyranosyl bromide (5). To a suspension of D-glucose $(4.55 \mathrm{~g}, 0.025 \mathrm{~mol})$ in pyridine $(50 \mathrm{~mL})$ were added $\operatorname{TrCl}(10.6 \mathrm{~g}, 0.038 \mathrm{~mol})$ and $\mathrm{Et}_{3} \mathrm{~N}(18 \mathrm{~mL}, 0.13 \mathrm{~mol})$, and the mixture was stirred at room temperature overnight. After the addition of $\mathrm{BzCl}(23 \mathrm{~mL}, 0.20 \mathrm{~mol})$ at the same temperature, stirring was continued overnight. The reaction was quenched with sat. aq. $\mathrm{NaHCO}_{3}$, and the mixture was extracted with $\mathrm{CHCl}_{3}$. The organic layer was dried $\left(\mathrm{Na}_{2} \mathrm{SO}_{4}\right)$, and concentrated in vacuo. After removal of the high polarity byproducts by silica gel short column (hexane/EtOAc $=5: 1$ ), a crude was used in the next step without further purification. The crude product was solved in $\mathrm{MeOH} / \mathrm{EtOAc}(1: 1,100 \mathrm{~mL})$. After the addition of $5 \%$ Pd-C (cat.), the mixture was stirred overnight under $\mathrm{H}_{2}$ atmosphere. The mixture was filtered and the filtrate was concentrated in vacuo. After silica gel short column chromatography (hexane/EtOAc $=3: 1$ ), a crude was used in the next step without further purification.

A mixture of the crude and FmocCl $(9.80 \mathrm{~g}, 0.038 \mathrm{~mol})$ in pyridine $(100 \mathrm{~mL})$ was stirred overnight. The reaction was quenched by sat. aq. $\mathrm{NaHCO}_{3}$, and the mixture was extracted with $\mathrm{CHCl}_{3}$. The organic layer was dried $\left(\mathrm{Na}_{2} \mathrm{SO}_{4}\right)$, and concentrated in vacuo. After purification by silica gel short column chromatography (hexane/EtOAc $=10: 1$ ), a crude was used in the next step without further purification. A mixture of the crude, $\mathrm{HBr}-\mathrm{AcOH}(250 \mathrm{~mL}), \mathrm{AcOH}(60 \mathrm{~mL})$, and $\mathrm{Ac}_{2} \mathrm{O}(60 \mathrm{~mL})$ was stirred overnight. The mixture was extracted with $\mathrm{CHCl}_{3}$. The organic layer was dried $\left(\mathrm{Na}_{2} \mathrm{SO}_{4}\right)$, and concentrated in vacuo. The residue was purified by silica gel column chromatography (hexane/EtOAc $=$ 5:1) to give 5 as a colorless oil $\left(8.43 \mathrm{~g}, 43 \%\right.$ in 4 steps): $[\alpha]^{23}{ }_{\mathrm{D}}+76.4\left(c 1.00, \mathrm{CHCl}_{3}\right)$; IR (film) 1732 , 1601, $1261 \mathrm{~cm}^{-1}$; ${ }^{1} \mathrm{H}-\mathrm{NMR}(400 \mathrm{MHz}) \delta 4.40$ (5H, m, H-6a,6b, Fmoc), 4.67 (1H, m, H-5), $5.36(1 \mathrm{H}$, dd, $J=9.8,4.0 \mathrm{~Hz}, \mathrm{H}-2), 5.78(1 \mathrm{H}, \mathrm{t}, J=9.8 \mathrm{~Hz}, \mathrm{H}-4), 6.28(1 \mathrm{H}, \mathrm{t}, J=9.8 \mathrm{~Hz}, \mathrm{H}-3), 6.89(1 \mathrm{H}, \mathrm{d}$, $J=4.0 \mathrm{~Hz}, \mathrm{H}-4), 7.40(11 \mathrm{H}, \mathrm{m}, \mathrm{Ar}), 7.54(2 \mathrm{H}, \mathrm{m}, \mathrm{Ar}), 7.64(2 \mathrm{H}, \mathrm{m}, \mathrm{Ar}), 7.78(2 \mathrm{H}, \mathrm{m}, \mathrm{Ar}), 7.89$ (2H, m, Ar), 8.00 (4H, m, Ar); ${ }^{13} \mathrm{C}-\mathrm{NMR}$ (100 MHz) $\delta 46.5$ (Fmoc), 64.9 (C-6), 67.7 (C-4), 70.4 (C-3), 70.5 (C-2), 71.3 (C-5), $72.5\left(\mathrm{FmocCH}_{2}\right), 86.7$ (C-1), 120.0 (Ar), 125.2 (Ar), 125.3 (Ar), 127.2 (Ar), 127.9 
(Ar), 128.3 (Ar), 128.4 (Ar), 128.5 (Ar), 128.7 (Ar), 129.7 (Ar), 129.9 (Ar), 130.0 (Ar), 133.3 (Ar), 133.7 (Ar), 133.85 (Ar), 141.2 (Ar), 141.3 (Ar), 143.2 (Ar), $143.4(\mathrm{Ar}), 154.7(\mathrm{C}=\mathrm{O}), 165.1(\mathrm{C}=\mathrm{O})$, $165.2(\mathrm{C}=\mathrm{O}), 165.5(\mathrm{C}=\mathrm{O})$. ESI-MS: calcd for $\mathrm{C}_{42} \mathrm{H}_{33} \mathrm{O}_{10} \mathrm{NaBr} 799.1155(\mathrm{M}+\mathrm{Na})^{+}$, found $\mathrm{m} / z$ 799.1151.

(2R,5R,6R, 7S)-5-(((((9H-Fluoren-9-yl)methoxy) carbonyl)oxy)methyl)-2-((2-((tert-butyldimethylsilyl)oxy)4-methoxybenzyl)oxy)-2-phenyltetrahydro-3aH-[1,3]dioxolo[4,5-b]pyran-6,7-diyl dibenzoate (2a) and 2-tert-Buthyldimethylsyloxy-4-methoxyphenylmethyl2,3,4-tetra-O-benzoyl-6-O-9-fluorenylmethyloxycarboxyl$\beta$-D-glucopyranoside (2b). To a solution of $5(71 \mathrm{mg}, 0.092 \mathrm{mmol})$ in anhydrous toluene $(0.9 \mathrm{~mL})$ were added $6(74 \mathrm{mg}, 0.27 \mathrm{mmol}), \mathrm{Hg}(\mathrm{CN})_{2}(46 \mathrm{mg}, 0.18 \mathrm{mmol})$, and $\mathrm{MS} 4 \mathrm{~A}$ at $100{ }^{\circ} \mathrm{C}$, and the mixture was stirred overnight. The mixture was filtered through a Celite pad, and the filtrate was washed with sat. aq. $\mathrm{NaHCO}_{3}$. The organic extracts were dried $\left(\mathrm{Na}_{2} \mathrm{SO}_{4}\right)$, and concentrated in vacuo. The residue was purified by silica gel column chromatography (hexane/acetone $=7: 1$ ) to give $\mathbf{2 a}$ (45 mg, 50\%) and $\mathbf{2 b}(26 \mathrm{mg}, 30 \%)$ as oil: $\mathbf{2 a}:[\alpha]^{23}{ }_{\mathrm{D}}+1.2\left(\mathrm{c} 1.00, \mathrm{CHCl}_{3}\right)$; IR (film) 1728, 1613, 1257 $\mathrm{cm}^{-1}$; ${ }^{1} \mathrm{H}-\mathrm{NMR}(400 \mathrm{MHz}) \delta 0.15\left(6 \mathrm{H}, \mathrm{s}, \mathrm{SiCH}_{3}\right), 0.93(9 \mathrm{H}, \mathrm{s}, \mathrm{tBu}), 3.74\left(3 \mathrm{H}, \mathrm{s}, \mathrm{OCH}_{3}\right), 4.16(2 \mathrm{H}, \mathrm{m}$, Fmoc), 4.29 (6H, m, H-5,6a,6b, $\left.\mathrm{ArCH}_{2}, \mathrm{Fmoc}\right), 4.78$ (1H, m, H-2), 5.37 (1H, bd, H-4), 5.76 (1H, br, H-3), $6.04(1 \mathrm{H}, \mathrm{bd}, \mathrm{H}-1), 6.31(1 \mathrm{H}, \mathrm{d}, J=2.2 \mathrm{~Hz}, \mathrm{Ar}), 6.48(1 \mathrm{H}, \mathrm{dd}, J=8.3,2.2 \mathrm{~Hz}, \mathrm{Ar}), 7.18(1 \mathrm{H}, \mathrm{d}$, $J=8.3 \mathrm{~Hz}, \mathrm{Ar}), 7.41$ (11H, m, Ar), 7.57 (4H, m, Ar), 7.75 (2H, m, Ar), 7.82 (2H, m, Ar), 7.97 (2H, m, $\mathrm{Ar}), 8.11(2 \mathrm{H}, \mathrm{m}, \mathrm{Ar}) ;{ }^{13} \mathrm{C}-\mathrm{NMR}(100 \mathrm{MHz}) \delta-4.3\left(\mathrm{SiCH}_{3}\right), 18.1(\mathrm{tBu}), 25.6(\mathrm{tBu}), 46.6(\mathrm{Fmoc}), 55.2$ $\left(\mathrm{OCH}_{3}\right), 61.3\left(\mathrm{ArCH}_{2}\right), 67.3(\mathrm{C}-6), 68.3(\mathrm{C}-2), 69.2(\mathrm{C}-3), 70.1(\mathrm{C}-4), 72.3\left(\mathrm{FmocCH}_{2}\right), 77.2(\mathrm{C}-5)$, 97.4 (C-1), 105.3 (Ar), 105.9 (Ar), 119.9 (Ar), 120.5 (Ar), 121.4 (Ar), 125.3 (orthoester-C), 126.5 (Ar), 127.1 (Ar), 127.2 (Ar), 127.8 (Ar), 128.3 (Ar), 1283.4 (Ar), 128.5 (Ar), 129.1 (Ar), 129.6 (Ar), 129.9 (Ar), 130.0 (Ar), 133.5 (Ar), 133.6 (Ar), 135.3 (Ar), 141.2 (Ar), 143.2 (Ar), 154.0 (C=O), 154.9 $(\mathrm{C}=\mathrm{O}), 159.9(\mathrm{C}=\mathrm{O}), 165.2(\mathrm{C}=\mathrm{O})$. ESI-MS: calcd for $\mathrm{C}_{56} \mathrm{H}_{56} \mathrm{O}_{13} \mathrm{SiNa} 987.3388(\mathrm{M}+\mathrm{Na})^{+}$, found $\mathrm{m} / \mathrm{z}$ 987.3373. 2b: $[\alpha]^{23}{ }_{\mathrm{D}}-14.9$ (c 1.00, $\mathrm{CHCl}_{3}$ ); IR (film) 1735, 1610, $1262 \mathrm{~cm}^{-1} ;{ }^{1} \mathrm{H}-\mathrm{NMR}(400 \mathrm{MHz}) \delta$ $0.10\left(3 \mathrm{H}, \mathrm{s}, \mathrm{SiCH}_{3}\right), 0.16\left(3 \mathrm{H}, \mathrm{s}, \mathrm{SiCH}_{3}\right), 0.94(9 \mathrm{H}, \mathrm{s}, \mathrm{tBu}), 3.72\left(3 \mathrm{H}, \mathrm{s}, \mathrm{OCH}_{3}\right), 4.06(1 \mathrm{H}, \mathrm{ddd}$, $J=9.8,5.3,3.2 \mathrm{~Hz}, \mathrm{H}-5), 4.23$ (1H, t, $J=7.6 \mathrm{~Hz}$, Fmoc), 4.40 (4H, m, H-6a,6b, Fmoc), 4.76 (1H, d, $\left.J=12.4 \mathrm{~Hz}, \mathrm{ArCH}_{2}\right), 4.83\left(1 \mathrm{H}, \mathrm{d}, J=12.4 \mathrm{~Hz}, \mathrm{ArCH}_{2}\right), 4.86(1 \mathrm{H}, \mathrm{d}, J=8.0 \mathrm{~Hz}, \mathrm{H}-1), 5.58(1 \mathrm{H}, \mathrm{dd}$, $J=9.8,8.0 \mathrm{~Hz}, \mathrm{H}-2), 5.60$ (1H, t, $J=9.8 \mathrm{~Hz}, \mathrm{H}-4), 5.83$ (1H, t, $J=9.8 \mathrm{~Hz}, \mathrm{H}-3), 6.27$ (1H, dd, $J=8.7$, $2.5 \mathrm{~Hz}, \mathrm{Ar}), 6.30(1 \mathrm{H}, \mathrm{d}, J=2.5 \mathrm{~Hz}, \mathrm{Ar}), 7.12(1 \mathrm{H}, \mathrm{d}, J=8.7 \mathrm{~Hz}, \mathrm{Ar}), 7.35(14 \mathrm{H}, \mathrm{m}, \mathrm{Ar}), 7.50(2 \mathrm{H}, \mathrm{m}$, Ar), $7.62(2 \mathrm{H}, \mathrm{m}, \mathrm{Ar}), 7.76(2 \mathrm{H}, \mathrm{m}, \mathrm{Ar}), 7.83(4 \mathrm{H}, \mathrm{m}, \mathrm{Ar}), 7.92(1 \mathrm{H}, \mathrm{m}, \mathrm{Ar}) ;{ }^{13} \mathrm{C}-\mathrm{NMR}(100 \mathrm{MHz}) \delta$ -4.4 $\left.\left(\mathrm{SiCH}_{3}\right),-4.3\left(\mathrm{SiCH}_{3}\right), 18.1(\mathrm{tBu}), 25.6(\mathrm{tBu}), 29.7(\mathrm{tBu}), 46.6(\mathrm{Fmoc}), 55.2 \mathrm{OCH}_{3}\right), 65.6$ $\left(\mathrm{ArCH}_{2}\right), 66.3$ (C-6), 69.6 (C-4), 70.2 (C-3), 71.6 (C-2), 72.0 (C-5), $72.9\left(\mathrm{FmocCH}_{2}\right), 99.1$ (C-1), 105.4 (Ar), 105.9 (Ar), 119.6 (Ar), 119.9 (Ar), 125.2 (Ar), 125.3 (Ar), 127.2 (Ar), 127.8 (Ar), 128.1 (Ar), 128.2 (Ar), 128.4 (Ar), 128.8 (Ar), 129.3 (Ar), 129.7 (Ar), 129.8 (Ar), 129.9 (Ar), 131.2 (Ar), 133.0 (Ar), 133.2 (Ar), 133.4 (Ar), 141.2 (Ar), 143.2 (Ar), 143.4 (Ar), $154.7(\mathrm{C}=\mathrm{O}), 154.9(\mathrm{C}=\mathrm{O})$, $160.2(\mathrm{C}=\mathrm{O}), 164.9(\mathrm{C}=\mathrm{O}), 165.2(\mathrm{C}=\mathrm{O}), 165.8(\mathrm{C}=\mathrm{O})$. ESI-MS: calcd for $\mathrm{C}_{56} \mathrm{H}_{56} \mathrm{O}_{13} \mathrm{SiNa} 987.3388$ $(\mathrm{M}+\mathrm{Na})^{+}$, found $\mathrm{m} / \mathrm{z} 987.3381$.

$(2 R, 5 R, 6 R, 7 S)-2-((2-(($ tert-Butyldimethylsilyl)oxy)-4-methoxybenzyl)oxy)-5-(hydroxymethyl)-2-phenyltetrahydro-3aH-[1,3]dioxolo[4,5-b]pyran-6,7-diyl dibenzoate (3). To a solution of 2a (30 $\mathrm{mg}$, $0.032 \mathrm{mmol})$ in pyridine $(0.5 \mathrm{~mL})$ was added $\mathrm{Et}_{3} \mathrm{~N}(13 \mu \mathrm{L}, 0.095 \mathrm{mmol})$ at room temperature. After being stirred overnight, the reaction mixture was concentrated in vacuo. The residue was purified by 
silica gel column chromatography (hexane/acetone $=2: 1$ ) to give $\mathbf{3}$ as a clear oil $(19 \mathrm{mg}, 81 \%)$ : $[\alpha]^{23} \mathrm{D}+9.5\left(\mathrm{c} 1.00, \mathrm{CHCl}_{3}\right)$; IR (film) 3527, 1725, 1613, $1261 \mathrm{~cm}^{-1} ;{ }^{1} \mathrm{H}-\mathrm{NMR}(400 \mathrm{MHz}) \delta 0.15(6 \mathrm{H}$, s, $\left.\mathrm{SiCH}_{3}\right), 0.90(9 \mathrm{H}, \mathrm{s}, \mathrm{tBu}), 3.65(1 \mathrm{H}, \mathrm{m}, \mathrm{H}-6), 3.75\left(3 \mathrm{H}, \mathrm{s}, \mathrm{OCH}_{3}\right), 3.78(1 \mathrm{H}, \mathrm{m}, \mathrm{H}-6), 3.89(1 \mathrm{H}, \mathrm{m}$, H-5), $4.29\left(1 \mathrm{H}, \mathrm{d}, J=11.2 \mathrm{~Hz}, \mathrm{ArCH}_{2}\right), 4.36\left(1 \mathrm{H}, \mathrm{d}, J=11.2 \mathrm{~Hz}, \mathrm{ArCH}_{2}\right), 4.75(1 \mathrm{H}, \mathrm{ddd}, J=5.2,2.9$, $0.8 \mathrm{~Hz}, \mathrm{H}-2), 5.37$ (1H, m, H-4), 5.74 (1H, m, H-3), 6.03 (1H, bd, H-1), 6.31 (1H, d, J=2.5 Hz, Ar), $6.48(1 \mathrm{H}, \mathrm{dd}, J=8.5,2.5 \mathrm{~Hz}, \mathrm{Ar}), 7.18(1 \mathrm{H}, \mathrm{d}, J=8.5 \mathrm{~Hz}, \mathrm{Ar}), 7.44$ (7H, m, Ar), 7.60 (2H, m, Ar), $7.81(2 \mathrm{H}, \mathrm{m}, \mathrm{Ar}), 7.98(2 \mathrm{H}, \mathrm{m}, \mathrm{Ar}), 8.05(2 \mathrm{H}, \mathrm{m}, \mathrm{Ar}) ;{ }^{13} \mathrm{C}-\mathrm{NMR}(100 \mathrm{MHz}) \delta-4.3\left(\mathrm{SiCH}_{3}\right), 18.1$ $(\mathrm{tBu}), 25.6(\mathrm{tBu}), 55.2\left(\mathrm{OCH}_{3}\right), 61.2\left(\mathrm{ArCH}_{2}\right), 62.4(\mathrm{C}-6), 68.3(\mathrm{C}-2), 69.4(\mathrm{C}-3), 69.8(\mathrm{C}-4), 72.6(\mathrm{C}-5)$, 97.5 (C-1), 105.3 (Ar), 105.9 (Ar), 120.5 (Ar), 121.1 (Ar), 126.4 (orthoester-C), 128.3 (Ar), 128.4 (Ar), 128.5 (Ar), 129.0 (Ar), 129.1 (Ar), 129.6 (Ar), 129.9 (Ar), 130.0 (Ar), 133.5 (Ar), 133.6 (Ar), 135.3 (Ar), $154.0(\mathrm{C}=\mathrm{O}), 159.9(\mathrm{C}=\mathrm{O}), 164.6(\mathrm{C}=\mathrm{O}), 165.6(\mathrm{C}=\mathrm{O})$. ESI-MS: calcd for $\mathrm{C}_{41} \mathrm{H}_{46} \mathrm{O}_{11} \mathrm{NaSi}$ $765.2707(\mathrm{M}+\mathrm{Na})^{+}$, found $m / z 765.2710$.

Cyclohexyl 2,3,4-tri-O-benzoyl-6-O-9-fluorenylmethyloxycarboxyl- $\beta$-D-glucopyranoside (7). A $0.1 \mathrm{M}$ solution of cyclohexanol in 1,2-DCE $(10 \mathrm{~mL})$ containing $4 \AA \mathrm{MS}$ and $\mathrm{Bu}_{4} \mathrm{NClO}_{4}(3.42 \mathrm{~g}, 1 \mathrm{M})$ as a supporting salt, was electrolyzed by constant current electrolysis (C.C.E., $6 \mathrm{~mA} / \mathrm{cm}^{2}$ ) at $40{ }^{\circ} \mathrm{C}$, using a glassy carbon plate $(1.5 \mathrm{~cm} \times 1.5 \mathrm{~cm})$ as an anode and a Pt plate $(1.8 \mathrm{~cm} \times 1.8 \mathrm{~cm})$ as a cathode. After the electrolysis, the reaction mixture $(3.4 \mathrm{~mL}, 0.1 \mathrm{M}$ EGA solution, 3 equiv.) was added to a solution of $2 \mathbf{a}(0.11 \mathrm{~g}, 0.11 \mathrm{mmol})$, cyclohexanol $(0.4 \mathrm{~mL}, 0.34 \mathrm{mmol})$, and $4 \AA$ MS in 1,2-DCE (1.1 mL). After being stirred $20 \mathrm{~min}$, the mixture was filtered through a Celite pad and the filtrate was washed with sat. aq. $\mathrm{NaHCO}_{3}$. The organic extracts were dried $\left(\mathrm{Na}_{2} \mathrm{SO}_{4}\right)$, and concentrated in vacuo. The residue was purified by preparative TLC (hexane/acetone $=5: 1)$ to give 7 as an oil $(0.056 \mathrm{~g}, 62 \%):[\alpha]_{\mathrm{D}}^{23}-4.10$ (c 1.00, $\mathrm{CHCl}_{3}$ ); IR (film) 1732, 1601, $1262 \mathrm{~cm}^{-1} ;{ }^{1} \mathrm{H}-\mathrm{NMR}(400 \mathrm{MHz}) \delta 1.22(5 \mathrm{H}, \mathrm{m}$, cyclohexyl), $1.46(2 \mathrm{H}, \mathrm{m}$, cyclohexyl), $1.70(2 \mathrm{H}, \mathrm{m}$, cyclohexyl), $1.88(1 \mathrm{H}, \mathrm{m}$, cyclohexyl), $3.72(1 \mathrm{H}, \mathrm{m}$, cyclohexyl), 4.09 (1H, m, Fmoc), 4.22 (1H, m, Fmoc), 4.36 (3H, m, H-5,6a, Fmoc), 4.46 (1H, d, $J=11.4,6.5 \mathrm{~Hz}, \mathrm{H}-6 \mathrm{~b}), 4.93(1 \mathrm{H}, \mathrm{d}, J=8.0 \mathrm{~Hz}, \mathrm{H}-1), 5.51(1 \mathrm{H}, \mathrm{dd}, J=9.6,8.0 \mathrm{~Hz}, \mathrm{H}-2), 5.55(1 \mathrm{H}, \mathrm{t}$, $J=9.6 \mathrm{~Hz}, \mathrm{H}-4), 5.90$ (1H, t, $J=9.6 \mathrm{~Hz}, \mathrm{H}-3), 7.30(11 \mathrm{H}, \mathrm{m}, \mathrm{Ar}), 7.51$ (2H, m, Ar), 7.60 (2H, m, Ar), 7.77 (2H, m, Ar), 7.84 (2H, m, Ar), 7.95 (4H, m, Ar); ${ }^{13} \mathrm{C}-\mathrm{NMR}(100 \mathrm{MHz}) \delta 23.4$ (cyclohexyl), 23.6 (cyclohexyl), 25.3 (cyclohexyl), 31.5 (cyclohexyl), 33.1 (cyclohexyl), 46.6 (Fmoc), 66.5 (C-6), 69.8 (C-4), 70.2 (C-2,3), 72.0 (FmocCH $), 72.9$ (C-5), 78.2 (cyclohexyl), 99.7 (C-1), 120.0 (Ar), 125.2 (Ar), 127.2 (Ar), 127.9 (Ar), 128.3 (Ar), 128.4 (Ar), 128.7 (Ar), 128.8 (Ar), 129.5 (Ar), 129.7 (Ar), 129.8 (Ar), 133.1 (Ar), 133.2 (Ar), 133.5 (Ar), 141.2 (Ar), 143.2 (Ar), 123.3 (Ar), $154.8(\mathrm{C}=\mathrm{O}), 164.9$ $(\mathrm{C}=\mathrm{O}), 165.3(\mathrm{C}=\mathrm{O}), 165.8(\mathrm{C}=\mathrm{O})$. ESI-MS: calcd for $\mathrm{C}_{48} \mathrm{H}_{45} \mathrm{O}_{11} 7997.2962(\mathrm{M}+\mathrm{H})^{+}$, found $\mathrm{m} / z$ 797.2936.

Cyclohexyl 2,3,4-tri-O-benzoyl- $\beta$-D-glucopyranoside (8). To a solution of 7 (54 $\mathrm{mg}, 0.068 \mathrm{mmol}$ ) in pyridine $(1 \mathrm{~mL})$ was added $\mathrm{Et}_{3} \mathrm{~N}(19 \mu \mathrm{L}, 0.14 \mathrm{mmol})$ at room temperature, and the mixture was stirred overnight. The mixture was concentrated in vacuo. The residue was purified by silica gel column chromatography (hexane/acetone $=2: 1)$ to give 8 as an oil $(37 \mathrm{mg}, 95 \%):[\alpha]^{23}{ }^{-11.5}\left(c 1.00, \mathrm{CHCl}_{3}\right)$; IR (film) 3523, 1731, 1601, $1263 \mathrm{~cm}^{-1} ;{ }^{1} \mathrm{H}-\mathrm{NMR}$ (400 MHz) $\delta 1.05(3 \mathrm{H}, \mathrm{m}$, cyclohexyl), $1.48(6 \mathrm{H}, \mathrm{m}$, cyclohexyl), 1.77 (1H, m, cyclohexyl), $2.41(1 \mathrm{H}, \mathrm{bs}, \mathrm{OH}), 3.68$ (4H, m, cyclohexyl, H-5,6a,6b), 4.38 $(1 \mathrm{H}, \mathrm{d}, J=8.0 \mathrm{~Hz}, \mathrm{H}-1), 5.37$ (2H, bt, H-2,4), $5.82(1 \mathrm{H}, \mathrm{t}, J=9.6 \mathrm{~Hz}, \mathrm{H}-3), 7.18$ (3H, m, Ar), 7.31 $(4 \mathrm{H}, \mathrm{m}, \mathrm{Ar}), 7.41(2 \mathrm{H}, \mathrm{m}, \mathrm{Ar}), 7.74(2 \mathrm{H}, \mathrm{m}, \mathrm{Ar}), 7.85(4 \mathrm{H}, \mathrm{m}, \mathrm{Ar}) ;{ }^{13} \mathrm{C}-\mathrm{NMR}(100 \mathrm{MHz}) \delta 23.4$ 
(cyclohexyl), 23.6 (cyclohexyl), 25.4 (cyclohexyl), 31.5 (cyclohexyl), 33.2 (cyclohexyl), 61.5 (C-6), 69.7 (C-4,4',6'), 72.1 (C-2,3,3'), 72.9 (C-2',5'), 74.5 (C-5), 78.1 (cyclohexyl), 99.6 (C-1,1'), 128.2 (Ar), 128.3 (Ar), 128.5 (Ar), 128.6 (Ar), 128.9 (Ar), 129.5 (Ar), 129.6 (Ar), 129.7 (Ar), 129.9 (Ar), 133.1 (Ar), 133.2 (Ar), $133.6(\mathrm{Ar}), 164.9(\mathrm{C}=\mathrm{O}), 165.8(\mathrm{C}=\mathrm{O}), 165.9(\mathrm{C}=\mathrm{O})$. ESI-MS: calcd for $\mathrm{C}_{33} \mathrm{H}_{34} \mathrm{O}_{9} \mathrm{Na}$ $597.2101(\mathrm{M}+\mathrm{Na})^{+}$, found $m / z 597.2112$.

Cyclohexyl 2,3,4-tri-O-benzoyl- $\beta$-D-glucopyranosyl-(1 $\rightarrow 6)$-2,3,4-tri-O-benzoyl-6-O-9-fluorenylmethyloxycarboxyl- $\beta$-D-glucopyranoside (9). A solution of cyclohexanol in 1,2-DCE (10 mL, $0.1 \mathrm{M})$ containing $4 \AA \mathrm{MS}$ and $\mathrm{Bu}_{4} \mathrm{NClO}_{4}(3.42 \mathrm{~g}, 1 \mathrm{M})$ as a supporting salt, was electrolyzed by constant current electrolysis (C.C.E., $\left.6 \mathrm{~mA} / \mathrm{cm}^{2}\right)$ at $40{ }^{\circ} \mathrm{C}$, using a glassy carbon plate $(1.5 \mathrm{~cm} \times 1.5 \mathrm{~cm})$ as an anode and a Pt plate $(1.8 \mathrm{~cm} \times 1.8 \mathrm{~cm})$ as a cathode. The reaction mixture $(0.6 \mathrm{~mL}, 0.1 \mathrm{M}$ EGA solution, 3 equiv.) was added to a solution of $\mathbf{2 a}(31 \mathrm{mg}, 0.032 \mathrm{mmol})$ and $\mathbf{8}(37 \mathrm{mg}, 0.064 \mathrm{mmol})$, and MS 4A in 1,2-DCE $(0.5 \mathrm{~mL})$. After being stirred $20 \mathrm{~min}$, the mixture was filtered through a Celite pad, and the filtrate was washed with sat. aq. $\mathrm{NaHCO}_{3}$. The organic extract was dried $\left(\mathrm{Na}_{2} \mathrm{SO}_{4}\right)$, and concentrated in vacuo. The residue was purified by preparative TLC (hexane/acetone $=2: 1$ ) to give 9 as an oil (16 mg, 38\%): $[\alpha]^{23}{ }_{\mathrm{D}}-9.8$ (c 1.00, $\mathrm{CHCl}_{3}$ ); IR (film) 1734, 1601, $1261 \mathrm{~cm}^{-1}$; ${ }^{1} \mathrm{H}-\mathrm{NMR}$ (400 MHz) $\delta 1.41(10 \mathrm{H}, \mathrm{m}$, cyclohexyl), 3.62 (1H, m, cyclohexyl), 4.00 (4H, m, Fmoc, H-5,5'), 4.29 $\left(5 \mathrm{H}, \mathrm{m}, \mathrm{H}-6 \mathrm{a}, 6 \mathrm{~b}, 6 \mathrm{a}^{\prime}, 6 \mathrm{~b}^{\prime}\right), 4.78\left(1 \mathrm{H}, \mathrm{d}, J=7.8 \mathrm{~Hz}, \mathrm{H}-1^{\prime}\right), 5.04(1 \mathrm{H}, \mathrm{d}, J=7.8 \mathrm{~Hz}, \mathrm{H}-1), 5.34(1 \mathrm{H}, \mathrm{t}$, $\left.J=9.8 \mathrm{~Hz}, \mathrm{H}-4^{\prime}\right), 5.41\left(1 \mathrm{H}, \mathrm{dd}, J=9.8,7.8 \mathrm{~Hz}, \mathrm{H}-2^{\prime}\right), 5.48(1 \mathrm{H}, \mathrm{dd}, J=9.8,7.8 \mathrm{~Hz}, \mathrm{H}-2), 5.52(1 \mathrm{H}, \mathrm{t}$, $J=9.8 \mathrm{~Hz}, \mathrm{H}-4), 5.80(1 \mathrm{H}, \mathrm{t}, J=9.8 \mathrm{~Hz}, \mathrm{H}-3$ '), $5.83(1 \mathrm{H}, \mathrm{t}, J=9.8 \mathrm{~Hz}, \mathrm{H}-3), 7.41(22 \mathrm{H}, \mathrm{m}, \mathrm{Ar}), 7.62$ $(2 \mathrm{H}, \mathrm{m}, \mathrm{Ar}), 7.78(6 \mathrm{H}, \mathrm{m}, \mathrm{Ar}), 7.92(8 \mathrm{H}, \mathrm{m}, \mathrm{Ar}) ;{ }^{13} \mathrm{C}-\mathrm{NMR}(100 \mathrm{MHz}) \delta 22.9$ (cyclohexyl), 23.2 (cyclohexyl), 25.4 (cyclohexyl), 31.2 (cyclohexyl), 32.9 (cyclohexyl), 46.6 (Fmoc), 66.0 (C-6), 68.3 (C-6'), 69.3(C-4), 69.9 (C-4'), 70.3 (C-3), 71.7 (C-2,3'), 71.9 (C-2'), 72.1 C-5'), 72.8 (C-5), 72.9 (cyclohexyl), 74.1 (FmocCH ${ }_{2}$ ), 99.4 (C-1'), 100.8 (C-1), 119.9 (Ar), 125.3 (Ar), 125.4 (Ar), 127.2 (Ar), 127.8 (Ar), 128.2 (Ar), 128.3 (Ar), 128.4 (Ar), 128.7 (Ar), 128.9 (Ar), 129.3 (Ar), 129.5 (Ar), 129.6 (Ar), 129.7 (Ar), 129.8 (Ar), 133.0 (Ar), 133.1 (Ar), 133.2 (Ar), 133.4 (Ar), 133.5 (Ar), 141.2 (Ar), 143.3 (Ar), $143.4(\operatorname{Ar}), 154.8(\mathrm{C}=\mathrm{O}), 164.9(\mathrm{C}=\mathrm{O}), 165.1(\mathrm{C}=\mathrm{O}), 165.2(\mathrm{C}=\mathrm{O}), 165.4(\mathrm{C}=\mathrm{O})$, 165.6 $(\mathrm{C}=\mathrm{O}), 165.7(\mathrm{C}=\mathrm{O})$. ESI-MS: calcd for $\mathrm{C}_{75} \mathrm{H}_{66} \mathrm{O}_{19} \mathrm{Na} 1293.4096(\mathrm{M}+\mathrm{Na})^{+}$, found $\mathrm{m} / z$ 1293.4078.

Phenyl 2,3,4-tri-O-benzoyl-6-O-9-fluorenylmethyloxycarboxyl- $\beta$-D-glucopyranosyl-(1 $\rightarrow 6)-2,3,4-t r i-O$ benzoyl-1-thio- $\beta$-D-glucopyranoside (11). A solution of cyclohexanol in 1,2-DCE $(10 \mathrm{~mL}, 0.1 \mathrm{M})$ containing $4 \AA \mathrm{MS}$ and $\mathrm{Bu}_{4} \mathrm{NClO}_{4}(3.42 \mathrm{~g}, 1 \mathrm{M})$ as a supporting salt was electrolyzed by constant current electrolysis (C.C.E., $\left.6 \mathrm{~mA} / \mathrm{cm}^{2}\right)$ at $40{ }^{\circ} \mathrm{C}$, using a glassy carbon plate $(1.5 \mathrm{~cm} \times 1.5 \mathrm{~cm})$ as an anode and a Pt plate $(1.8 \mathrm{~cm} \times 1.8 \mathrm{~cm})$ as a cathode. After the electrolysis, the reaction mixture (1.3 mL, 0.1 M EGA solution, 3 equiv.) was added to a solution of $\mathbf{2 a}(34 \mathrm{mg}, 0.036 \mathrm{mmol}), \mathbf{1 0}$ (42 $\mathrm{mg}$, $0.072 \mathrm{mmol}), \mathrm{m}$ and $4 \AA \mathrm{MS}$ in 1,2-DCE $(0.5 \mathrm{~mL})$. After being stirred for $20 \mathrm{~min}$, the reaction mixture was filtered through a Celite pad, and the filtrate was washed with sat. aq. $\mathrm{NaHCO}_{3}$. The organic extracts were dried $\left(\mathrm{Na}_{2} \mathrm{SO}_{4}\right)$, and concentrated in vacuo. The residue was purified by preparative TLC (hexane/acetone $=2: 1)$ to give 11 as an oil $(29 \mathrm{mg}, 63 \%):[\alpha]^{23}{ }_{\mathrm{D}}+8.9\left(\mathrm{c} 1.00, \mathrm{CHCl}_{3}\right)$; IR (film) 1733 , 1601, $1261 \mathrm{~cm}^{-1} ;{ }^{1} \mathrm{H}-\mathrm{NMR}(400 \mathrm{MHz}) \delta 3.99$ (4H, m, Fmoc, H-5,5'), 4.24 (1H, m, Fmoc), 4.38 (4H, m, H-6a,6b,6a',6b'), $4.92\left(1 \mathrm{H}, \mathrm{d}, J=9.8 \mathrm{~Hz}, \mathrm{H}-1^{\prime}\right), 4.97(1 \mathrm{H}, \mathrm{d}, J=8.0 \mathrm{~Hz}, \mathrm{H}-1), 5.31(1 \mathrm{H}, \mathrm{t}$, $\left.J=9.8 \mathrm{~Hz}, \mathrm{H}-4^{\prime}\right), 5.34\left(1 \mathrm{H}, \mathrm{t}, J=9.8 \mathrm{~Hz}, \mathrm{H}-3^{\prime}\right), 5.51(2 \mathrm{H}, \mathrm{m}, \mathrm{H}-2,4), 5.81\left(1 \mathrm{H}, \mathrm{t}, J=9.8 \mathrm{~Hz}, \mathrm{H}-3^{\prime}\right)$, 
$5.85(1 \mathrm{H}, \mathrm{t}, J=9.8 \mathrm{~Hz}, \mathrm{H}-3), 7.23(2 \mathrm{H}, \mathrm{m}, \mathrm{Ar}), 7.39$ (25H, m, Ar), 7.61 (2H, m, Ar), 7.74 (4H, m, Ar), 7.85 (4H, m, Ar), 7.93 (6H, m, Ar); ${ }^{13} \mathrm{C}-\mathrm{NMR}$ (100 MHz) $\delta 46.6$ (Fmoc), 65.9 (C-6), $68.4(\mathrm{C}-6$ '), 69.4 (C-4'), 69.5 (C-4'), 70.2 (C-3'), 70.4 (C-3), 71.6 (C-2), 72.0 (C-2'), 72.8 (C-5'), $74.0\left(\mathrm{FmocCH}_{2}\right), 78.3$ (C-5), 85.9 (C-1'), 101.0 (C-1), 119.9 (Ar), 125.3 (Ar), 125.4 (Ar), 127.2 (Ar), 128.2 (Ar), 128.3 (Ar), 128.4 (Ar), 128.5 (Ar), 128.6 (Ar), 128.7 (Ar), 128.8 (Ar), 129.1 (Ar), 129.2 (Ar), 129.7 (Ar), 129.8 (Ar), 131.7 (Ar), 133.2 (Ar), 133.4 (Ar), 133.5 (Ar), 141.2 (Ar), 141.3 (Ar), 143.3 (Ar), 143.4 (Ar), $154.8(\mathrm{C}=\mathrm{O}), 164.9(\mathrm{C}=\mathrm{O}), 165.1(\mathrm{C}=\mathrm{O}), 165.2(\mathrm{C}=\mathrm{O}), 165.3(\mathrm{C}=\mathrm{O}), 165.6(\mathrm{C}=\mathrm{O}), 165.7(\mathrm{C}=\mathrm{O})$. ESI-MS: calcd for $\mathrm{C}_{75} \mathrm{H}_{60} \mathrm{O}_{18} \mathrm{NaS} 1303.3398(\mathrm{M}+\mathrm{Na})^{+}$, found $m / z$ 1303.3385.

Phenyl 2,3,4-tri-O-benzoyl-6-O-9-fluorenylmethyloxycarboxyl- $\beta$-D-glucopyranosyl-( $1 \rightarrow 6)-2,3,4-t r i-O$ benzoyl- $\beta$-D-glucopyranosyl- $(1 \rightarrow 6)$-2,3,4-tri-O-benzoyl-1-thio- $\beta$-D-glucopyranoside $\quad(\mathbf{1 2}) . \quad$ To a solution of $11(29 \mathrm{mg}, 0.022 \mathrm{mmol})$ in pyridine $(0.5 \mathrm{~mL})$ was added $\mathrm{Et}_{3} \mathrm{~N}(6.3 \mu \mathrm{L}, 0.045 \mathrm{mmol})$ at room temperature. After being stirred overnight, the mixture was concentrated in vacuo. The residue was purified by silica gel column chromatography (hexane/acetone $=2: 1$ ) to give an alcohol as an oil (19 mg, 82\%): $[\alpha]^{23}{ }_{\mathrm{D}}-2.1$ (c 1.00, $\mathrm{CHCl}_{3}$ ); IR (film) 3062, 1731, 1601, $1262 \mathrm{~cm}^{-1}$; ${ }^{1} \mathrm{H}-\mathrm{NMR}$ (400 MHz) $\delta 2.81(1 \mathrm{H}, \mathrm{dd}, J=8.0,5.6 \mathrm{~Hz}, \mathrm{OH}), 3.60(1 \mathrm{H}, \mathrm{m}, \mathrm{H}-5), 3.74(2 \mathrm{H}, \mathrm{m}, \mathrm{H}-5$ ',6a'), $3.95(1 \mathrm{H}$, dd, $\left.J=10.5,5.6 \mathrm{~Hz}, \mathrm{H}-6 \mathrm{~b}^{\prime}\right), 4.05$ (2H, m, H-6a,6b), $4.92\left(2 \mathrm{H}, \mathrm{bt}, \mathrm{H}-1,1^{\prime}\right), 5.23$ (1H, t, $J=9.6 \mathrm{~Hz}$, H-4'), $5.40\left(3 \mathrm{H}, \mathrm{m}, \mathrm{H}-2,2^{\prime}\right), 5.81(1 \mathrm{H}, \mathrm{t}, J=9.6 \mathrm{~Hz}, \mathrm{H}-3$ '), $5.85(1 \mathrm{H}, \mathrm{t}, J=9.6 \mathrm{~Hz}, \mathrm{H}-3), 7.38(19 \mathrm{H}, \mathrm{m}$, Ar), $7.53(4 \mathrm{H}, \mathrm{m}, \mathrm{Ar}), 7.76(2 \mathrm{H}, \mathrm{m}, \mathrm{Ar}), 7.81(2 \mathrm{H}, \mathrm{m}, \mathrm{Ar}), 7.93(8 \mathrm{H}, \mathrm{m}, \mathrm{Ar}) ;{ }^{13} \mathrm{C}-\mathrm{NMR}(100 \mathrm{MHz}) \delta$ 61.2 (C-6), 67.9 (C-6'), 69.4 (C-4'), 69.9 (C-4), 70.4 (C-3'), 71.5 (C-3), 72.8 (C-2), 73.9 (C-2'), 74.6 (C-5'), 77.8 (C-5), 86.0 (C-1'), 100.5 (C-1), 128.2 (Ar), 128.3 (Ar), 128.4 (Ar), 128.5 (Ar), 128.7 (Ar), 128.8 (Ar), 129.1 (Ar), 129.2 (Ar), 129.7 (Ar), 129.8 (Ar), 129.9 (Ar), 131.5 (Ar), 133.2 (Ar), 133.3 (Ar), 133.5 (Ar), $133.6(\operatorname{Ar}), 164.9(\mathrm{C}=\mathrm{O}), 165.1(\mathrm{C}=\mathrm{O}), 165.6(\mathrm{C}=\mathrm{O}), 165.7(\mathrm{C}=\mathrm{O}), 165.9(\mathrm{C}=\mathrm{O})$. ESI-MS: calcd for $\mathrm{C}_{60} \mathrm{H}_{50} \mathrm{O}_{16} \mathrm{NaS} 1081.2717(\mathrm{M}+\mathrm{Na})^{+}$, found $m / z$ 1081.2732.

A solution of cyclohexanol in 1,2-DCE $(10 \mathrm{~mL}, 0.1 \mathrm{M})$ containing $4 \AA \mathrm{MS}$ and $\mathrm{Bu}_{4} \mathrm{NClO}_{4}(3.42 \mathrm{~g}, 1 \mathrm{M})$ as a supporting salt, was electrolyzed by constant current electrolysis (C.C.E., $6 \mathrm{~mA} / \mathrm{cm}^{2}$ ) at $40{ }^{\circ} \mathrm{C}$, using a glassy carbon plate $(1.5 \mathrm{~cm} \times 1.5 \mathrm{~cm})$ as an anode and a Pt plate $(1.8 \mathrm{~cm} \times 1.8 \mathrm{~cm})$ as a cathode. The reaction mixture ( $0.6 \mathrm{~mL}, 0.1 \mathrm{M}$ EGA solution, 3 equiv. $)$ was added to a solution of $2 \mathrm{a}$ (37 mg, $0.038 \mathrm{mmol})$, the alcohol $(20 \mathrm{mg}, 0.019 \mathrm{mmol})$, and $4 \AA \mathrm{MS}$ in 1,2-DCE $(0.5 \mathrm{~mL})$. After being stirred at $40{ }^{\circ} \mathrm{C}$ for $20 \mathrm{~min}$, the reaction mixture was filtered through a Celite pad, and the filtrate was washed with saturated aqueous $\mathrm{NaHCO}_{3}$. The organic extracts were dried $\left(\mathrm{Na}_{2} \mathrm{SO}_{4}\right)$, and concentrated in vacuo. The residue was purified by preparative TLC $\left(\mathrm{Et}_{2} \mathrm{O} /\right.$ hexane $\left.=2: 1\right)$ to give $\mathbf{1 2}$ as a clear oil (4.1 mg, 12\%): $[\alpha]^{23}-5.4\left(c 1.00, \mathrm{CHCl}_{3}\right)$; IR (film) 1733, 1601, $1261 \mathrm{~cm}^{-1} ;{ }^{1} \mathrm{H}-\mathrm{NMR}(400 \mathrm{MHz}) \delta$ $3.62(1 \mathrm{H}, \mathrm{dd}, J=11.2,5.0 \mathrm{~Hz}, \mathrm{H}-6 \mathrm{a}), 3.84$ (3H, m, Fmoc, H-5',6), 4.01 (2H, m, H-5,5"), 4.31 (6H, m, H-6a,6b,6a',6b'), $4.61\left(1 \mathrm{H}, \mathrm{d}, J=8.0 \mathrm{~Hz}, \mathrm{H}-1^{\prime}\right), 4.99\left(1 \mathrm{H}, \mathrm{d}, J=10.0 \mathrm{~Hz}, \mathrm{H}-1^{\prime \prime}\right), 5.08(1 \mathrm{H}, \mathrm{d}$, $J=8.0 \mathrm{~Hz}, \mathrm{H}-1), 5.10(1 \mathrm{H}, \mathrm{t}, J=10.0 \mathrm{~Hz}, \mathrm{H}-4), 5.21\left(1 \mathrm{H}, \mathrm{dd}, J=9.8,8.0 \mathrm{~Hz}, \mathrm{H}-2^{\prime \prime}\right), 5.51(4 \mathrm{H}, \mathrm{m}$, H-2,2',4',4"), 5.68 (1H, t, $J=9.8$ Hz, H-3'), 5.84 (1H, t, $J=9.8 \mathrm{~Hz}, \mathrm{H}-3 "), 6.06$ (1H, t, $J=9.8 \mathrm{~Hz}, \mathrm{H}-3)$, $7.18(2 \mathrm{H}, \mathrm{m}, \mathrm{Ar}), 7.36(34 \mathrm{H}, \mathrm{m}, \mathrm{Ar}), 7.58(2 \mathrm{H}, \mathrm{m}, \mathrm{Ar}), 7.76(2 \mathrm{H}, \mathrm{m}, \mathrm{Ar}), 7.92(14 \mathrm{H}, \mathrm{m}, \mathrm{Ar}) ;{ }^{13} \mathrm{C}-\mathrm{NMR}$ (100 MHz) $\delta 46.6$ (Fmoc), 66.2 (C-6), 68.3 (C-6"), 69.4 (C-6'), 69.7 (C-4"), 70.1 (C-4), 70.2 (C-4'), 70.5 (C-3"), 71.7 (C-3), 71.9 (C-2,2',3'), 72.0 (C-2"), 72.6 (C-5'), 72.7 (C-5"), 73.9 (C-5), 74.1 ((FmocCH $\left.\mathrm{F}_{2}\right), 86.3$ (C-1"), 100.5 (C-1), 101.2 (C-1'), 119.9 (Ar), 120.0 (Ar), 125.3 (Ar), 125.4 (Ar), 
127.2 (Ar), 127.8 (Ar), 128.1 (Ar), 128.2 (Ar), 128.3 (Ar), 128.4 (Ar), 128.5 (Ar), 128.6 (Ar), 128.7 (Ar), 128.8 (Ar), 129.0 (Ar), 129.1 (Ar), 129.3 (Ar), 129.4 (Ar), 129.7 (Ar), 129.8 (Ar), 130.0 (Ar), 132.1 (Ar), 132.8 (Ar), 133.0 (Ar), 133.2 (Ar), 133.4 (Ar), 141.2 (Ar), 143.3 (Ar), 143.4 (Ar), 154.8 $(\mathrm{C}=\mathrm{O}), 164.9(\mathrm{C}=\mathrm{O}), 165.2(\mathrm{C}=\mathrm{O}), 165.3(\mathrm{C}=\mathrm{O}), 165.7(\mathrm{C}=\mathrm{O})$. ESI-MS: calcd for $\mathrm{C}_{102} \mathrm{H}_{82} \mathrm{O}_{26} \mathrm{NaS}$ $1777.4713(\mathrm{M}+\mathrm{Na})^{+}$, found $m / z$ 1777.4736.

\section{Conclusions}

The orthoester 2a bearing the selectively removable Fmoc group at the C-6 position was synthesized, and its utility as a glycosyl donor and acceptor after deprotection of the Fmoc group was demonstrated.

\section{Acknowledgments}

We thanked for MEXT-Supported Programs for the Strategic Research Foundation at Private Universities. 2009-2013, 2012-2016; Scientific Research (C) from MEXT; Sasakawa Research Grant for Financial Support (to KK).

\section{Author Contributions}

The listed authors contributed to this work as described in the following. Kohei Kawa synthesized the sugar derivatives. Tsuyoshi Saitoh made technical supports of the chemical and electrochemical procedures. Eisuke Kaji contributed with discussions of carbohydrate chemistry and the research direction. Shigeru Nishiyama conducted the research progress. All authors helped preparing manuscript and approved the final version.

\section{Conflicts of Interest}

The authors declare no conflict of interest.

\section{References and Notes}

1. Gantt, R.W.; Peltier-Pain, P.; Thorson, J.S. Enzymatic methods for glyco(diversification/randomization) of drugs and small molecules. Nat. Prod. Rep. 2011, 28, 1811-1853.

2. Toshima, K. Chemical biology based on target-selective degradation of proteins and carbohydrates using light-activatable organic molecules. Mol. Biosyst. 2013, 9, 834-854.

3. Toshima, K.; Tatsuta, K. Recent Progress in $O$-Glycosylation Methods and Its Application to Natural Products Synthesis. Chem. Rev. 1993, 93, 1503-1531.

4. Jacobsson, M.; Malmberg, J.; Ellervik, U. Aromatic O-glycosylation. Carbohydr. Res. 2006, 341, 1266-1281.

5. Stallforth, P.; Lepenies, B.; Adibekian, A.; Seeberger, P.H. Carbohydrates: A frontier in medicinal chemistry. J. Med. Chem. 2009, 52, 5561-5577.

6. Liptak, A.; Borbas, A.; Bajza, I. Protective Group Manipulation in Carbohydrate Synthesis. In Comprehensive Glycosciences; Kamering, J.P., Boons, G.-J., Lee, Y.C., Suzuki, A., Taniguchi, N., Vorangen, A.G.J., Eds.; Elsevier: Oxford, UK, 2007; pp. 203-259. 
7. Kochetkov, N.K.; Bochkov, A.F.; Sokolovskaya, T.A.; Snyatkova, V.J. Modifications of the orthoester method of glycosylation. Carbohydr. Res. 1971, 16, 17-27.

8. Bochkov, A.F.; Kochetkov, N.K. A new approach to the synthesis of oligosaccharides. Carbohydr. Res. 1975, 39, 355-357.

9. Bataneli, V.I.; Ovchinnikov, M.V.; Backinowsky, L.V.; Kochetkov, N.K. Glycosylation by 1,2-O-cyanothylidene derivatives of carbohydrates. Carbohydr. Res. 1979, 76, 252-256.

10. Kochetkov, N.K.; Nepogod'ev, S.A.; Backinowsky, L.V. Synthesis of cyclo-[(1-6)- $\beta-D-$ galactofurano]-oligosaccharides. Tetrahedron 1990, 46, 139-150.

11. Backinowaky, L.V.; Tavetkov, Y.E.; Balan, N.F.; Byramova, N.E.; Kochetkov, N.K. Synthesis of 1,2-trans-disaccharides via sugar thio-orthoesters. Carbohydr. Res. 1980, 85, 209-221.

12. Kunz, H.; Harreus, A. Glycosidsynthese mit 2,3,4,6-tetra- $O$-pivaloyl- $\alpha$-D-glucopyrancsylbromid. Liebigs Ann. Chem. 1982, 1982, 41-48.

13. Kunz, H.; Pfrengle, W. Effective 1,2-trans-glycosylation of complex alcohols and phenols using the oximateorthoester of $O$-pivaloyl glucopyranose. Chem. Commun. 1986, 713-714.

14. Kawa, K.; Saitoh, T.; Kaji, E.; Nishiyama, S. Development of glycosylation using the glucopyranose 1,2-orthobenzoate under electrochemical conditions. Org. Lett. 2013, 15, 5484-5487.

15. EGA was discussed in several papers [16-20].Upon comparison of methods using EGA, the case using EGA after preparation by anodic oxidation, was preferable to the simultaneous EGA-preparation and glycosylation [14]. A lower oxidation potential of cyclohexanol compared with the orthoester derivative in the reaction mixture, was effectively used for the abstraction of proton.

16. Uneyama, K. Electrochemistry 1; Steckhan, E., Ed.; Springer: Berlin/Heidelberg, Germany, 1987; Volume 142, pp. 167-188.

17. Torii, S. Electroorganic Synthesis, Part 1, Oxidation Methods, Applications; VerlagChemie: Weinheim, Germany, 1985; pp. 188-192.

18. Uneyama, K.; Ishimura, A.; Torii, S. Electrogenerated acid-catalyzed cyclization of isoprenoids. Bull. Chem. Soc. Jpn. 1985, 58, 1859-1860.

19. Nielsen, M.F. Encyclopedia of Electrochemistry; Shäfer, H.J., Ed.; Wiley-VHC: Weinheim, Germany, 2004; Volume 8, pp. 451-488.

20. Matsuo, K.; Shimazaki, H.; Sanada, T.; Shimada, K.; Hagiwara, S.; Suga, S.; Kashimura, S.; Yoshida, J. Electrogenerated acid (EGA)-catalyzed addition of diaryl disulfides to carbon-carbon multiple bonds. Chem. Lett. 2013, 42, 843-845.

21. When the glycosylation using $\mathrm{Ag}_{2} \mathrm{CO}_{3}$, the yield of $\mathbf{2 a}$ was reduced to $17 \%$.

22. The reaction condition has not yet optimized.

Sample Availability: Samples are available from the authors.

(C) 2014 by the authors; licensee MDPI, Basel, Switzerland. This article is an open access article distributed under the terms and conditions of the Creative Commons Attribution license (http://creativecommons.org/licenses/by/3.0/). 